\title{
Preface to Special Issue on SAT/CP Integration
}

\author{
Youssef Hamadi \\ Lucas Bordeaux \\ Microsoft Research \\ $7 \mathrm{~J} J$ Thomson Avenue \\ CB3 OFB Cambridge \\ United Kingdom
}

youssefh@microsoft. com
lucasb@microsoft.com

The techniques used in SAT (watched literals, activity-based heuristics, conflict analysis, restarts...) constitute a very successful combination that makes modern DPLL solvers amazingly robust. An interesting perspective for Constraint Programming $(\mathrm{CP})$ is to exploit and adapt these techniques in order to develop more powerful and easier-to-use solvers. One limitation of SAT, on the other hand, is that not all problems are effectively expressed in a Boolean format.

SAT and CP have many similarities, but also respective strengths and drawbacks. They can greatly benefit from each other. Based on these observations we organized the first workshop on the Integration of SAT and CP techniques as a satellite event of the twelfth International Conference on Principles and Practice of Constraint programming $\left(\mathrm{CP}^{\prime} \mathrm{O}\right)$ ).

Following the workshop announcement, the editor-in-chief of JSAT invited us to edit a special issue on the same topic of Integration of $S A T$ and $C P$ techniques. The call for papers was open and the submissions included both extended versions of papers presented at the workshop and new contributions. Four papers were finally selected.

The first two papers are successful integrations of ideas originating from SAT into the context of Constraint Satisfaction Problems:

- Recording and Minimizing Nogood from Restarts, by Christophe Lecoutre, Lakhdar Saïs, Sebastien Tabary, and Vincent Vidal, builds on the SAT-inspired themes of restarts and no-goods and shows how in the CSP context, nogoods can be learnt when a restart occurs, in order to avoid future explorations of a part of the search space already visited. This contribution extends a paper presented at IJCAI 07 by adding a nogood minimization technique.

- Using SAT Encodings to Derive CSP Value Ordering Heuristics, by Christophe Lecoutre, Lakhdar Saïs and Julien Vion, takes inspiration from SAT for another aspect of CSP, namely heuristics, and adapts in particular heuristics like Jeroslow-Wang.

The last two papers bring us closer to Satisfiability Modulo Theories, in which SAT solvers are extended with the ability to solve constraints over specific, non-Boolean domains, for instance the reals or the integers:

- Interpolant based Decision Procedure for Quantifier-Free Presburger Arithmetic, by Shuvendu K. Lahiri and Krishna K. Mehra, combines over- and under-approximation 
approaches of a formula, where Boolean interpolants are used to compute the overapproximation.

- Efficient Solving of Large Non-linear Arithmetic Constraint Systems with Complex Boolean Structure, by Martin Fränzle, Christian Herde, Stefan Ratschan, Tobias Schubert, and Tino Teige, reports on a fine-grained integration between real-valued interval arithmetics and Boolean reasoning, a combination of techniques particularly useful when reasoning on certain hybrid systems.

We would like to thank all the anonymous reviewers for their work, the authors for their contribution, and Hans van Maaren for giving us this opportunity of publishing this special issue. 